\title{
Harmonic terahertz gyrotron with quasi-optical confocal cavity
}

\author{
Wenjie Fu, Xiaotong Guan, Yang Yan, Xiaoyun Li, Yin Huang, and Lin Meng \\ School of Physical Electronics, University of Electronic Science and Technology of China, \\ Chengdu, 610054, China, fuwenjie@uestc.edu.cn
}

To develop terahertz gyrotron with low magnet, harmonic gyrotron is an interesting research area in terahertz gyrotron research area. However, in harmonic gyrotron, mode competition is more terrible than in fundamental gyrotron. Thus, adopting good mode-selectivity structures is a possible approach to improve harmonic numbers. Confocal waveguide is a type of quasi-optical open waveguide, which performs a much lower mode density than conventional cylindrical waveguide, and it is potential to be operated at high order mode with high frequency and high power. The confocal cavity utilized in gyrotron was initially proposed by R. J. Temkin et al. [1, 2], and it has been successfully used in $140 \mathrm{GHz}$ fundamental gyrotron oscillator and travelling wave amplifier in MIT. Due to the good mode selective characteristics, confocal waveguide would greatly suppress the mode competition in harmonic gyrotron. In University of Electronic Science and Technology of China (UESTC), the harmonic terahertz gyrotron with quasi-optical confocal cavity is being carried on, and a $400 \mathrm{GHz}$ second harmonic gyro-tron with confocal cavity is designed and experimented.

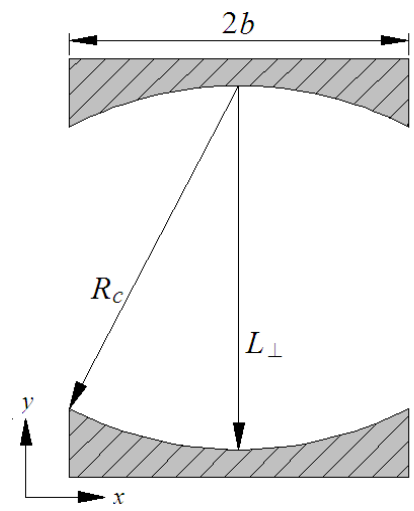

Fig. 1. Transverse geometry of confocal cylindrical waveguide

The cross section of a confocal waveguide is shown as Fig. 1. There are two cylindrical mirrors with each having a curvature radius $R_{c}$ separated by distance $L_{\perp}$, which is same length as $R_{c}$. Propagation modes in this confocal waveguide are TE and TM modes which are similar to that in cylindrical waveguide. Previous studies show that diffraction losses of $\mathrm{TE}_{m n}(m \neq 0)$ modes are greatly larger than that of $\mathrm{TE}_{0 n}$ modes, and only $\mathrm{TE}_{0 n}$ modes could be propagated in confocal waveguide. For $\mathrm{TE}_{0 n}$ mode, eigenvalue of wavenumber is given by

$$
k_{\perp, 0 n}=\left(n+\frac{1}{4}\right) \frac{\pi}{L_{\perp}}
$$

The frequency isolation between neighboring modes in confocal waveguide is $\Delta f=c / 2 L_{\perp}$, which is much higher than in cylinder waveguide. Thus, the quasioptical confocal waveguide has better mode selection, which greatly reduces the mode density. Equation (1) shows that $\mathrm{TE}_{0, s \times n-1}(s)$ mode has the maximum isolation from neighboring fundamental and lower harmonic modes. Therefore, the $\mathrm{TE}_{0, s \times n-1}(s)$ mode is potentially to be the operation mode at $s$ th harmonic. In $400 \mathrm{GHz}$ confocal gyrotron design, $\mathrm{TE}_{0,11}$ mode is chosen as the operation mode for second harmonic operation, and the eigenvalue characteristics of of $\mathrm{TE}_{0,11}$ with potential competition modes are shown in Fig. 2.
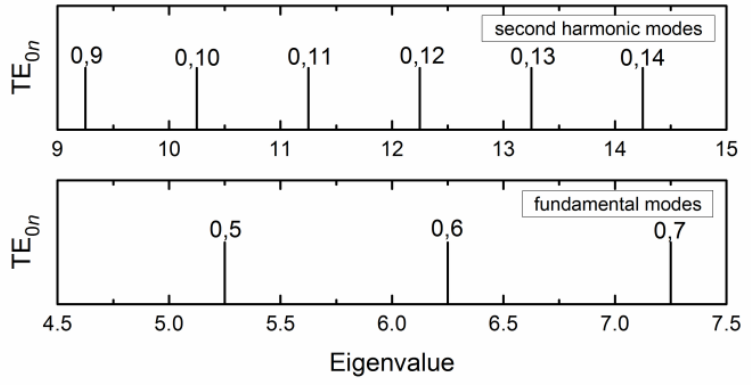

Fig. 2. Mode spectra in confocal waveguide

The confocal waveguide is a quasi-optical structure, some part of power will be diffracted out over the finite mirror aperture $2 b$. Thus, different from cylindrical cavity, the confocal cavity contains Ohmic loss, axial diffraction loss and transverse diffraction loss. The total $Q$ factor for $\mathrm{TE}_{0 n}$ modes in cavity could be expressed as

$$
\frac{1}{Q}=\frac{1}{Q_{\perp \text { diff }}}+\frac{1}{Q_{\text {zdiff }}}+\frac{1}{Q_{\text {ohmic }}}
$$

This denotes that the $Q$ value could be changed by adjusting the mirror aperture. Figure 3(a) displays the relationship between the diffraction loss rate and the mirror aperture. Figure 3(b) plots the calculated total $Q$ factor as a function of mirror aperture $2 a$ for different modes. In the design, the mirror aperture is selected to be $3.2 \mathrm{~mm}$ in which $\mathrm{TE}_{0,11}$ mode has a smaller diffraction loss and a suitable $Q$ factor.
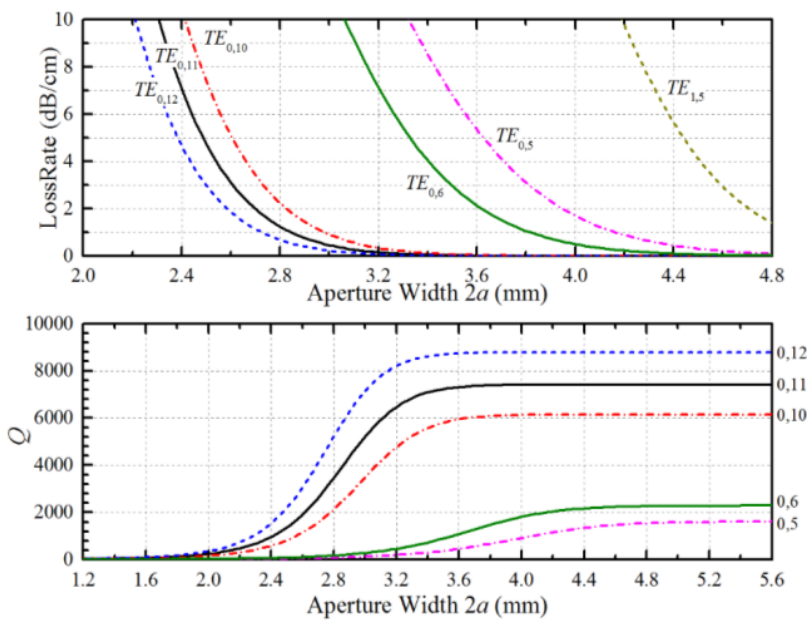

Fig. 3. Loss rate and $Q_{d i f f}$ versus mirror aperture $2 a$ 
Figure 4 displays the dependence of starting currents $I_{s t}$ for different modes and the magnetic field $B_{0}$, which calculated by linear theory of gyrotron. The results suggests that quasi-optical cavity performs much fewer competition modes within about $2 \mathrm{~T}$ magnetic operation range. The potential operation region of $\mathrm{TE}_{0,11}$ mode presents a large separation from that of fundamental $\mathrm{TE}_{0,5}$ and $\mathrm{TE}_{0,6}$ modes. While the operation magnetic field for $\mathrm{TE}_{0,5^{-}}$ $\mathrm{TE}_{0,10}$ and $\mathrm{TE}_{0,6}-\mathrm{TE}_{0,12}$ modes are close to each other, since these mode couples share a similar fundamental frequency of electron cyclotron. It is a good agreement with the above analysis of mode selection. Additionally, the operation regions of second harmonic modes are narrower than that of fundamental cyclotron, which implies that high harmonic can be excited only under strictly conditions.

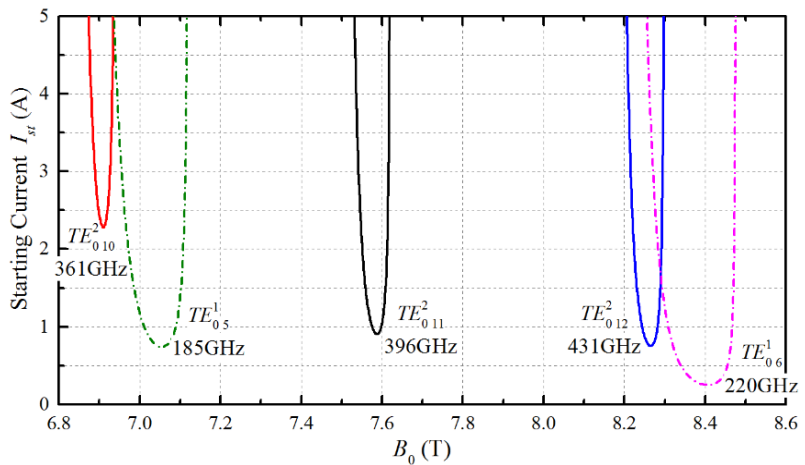

Fig. 4. Starting current $I_{s t}$ as function of magnetic field $B_{0}$

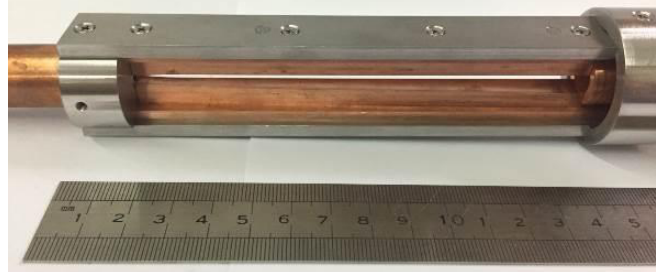

Fig. 5. Photo of confocal cavity

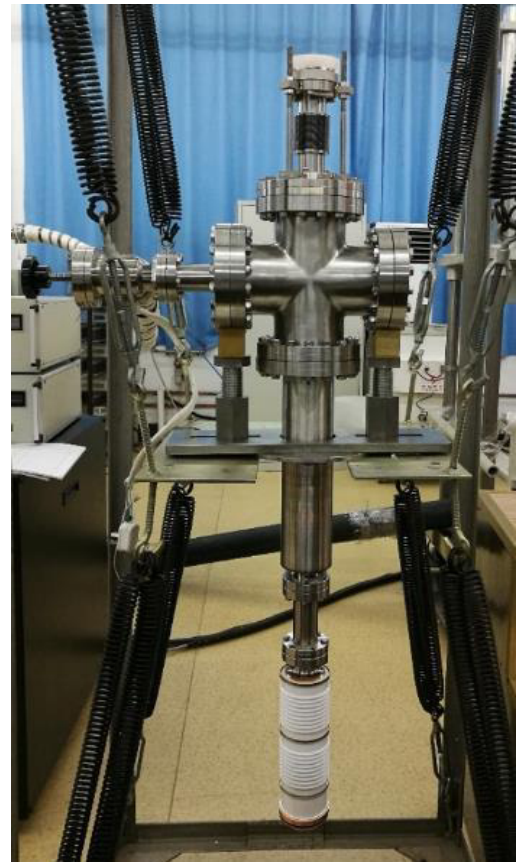

Fig. 6. Photo of $400 \mathrm{GHz}$ second harmonic prototype gyrotron with confocal cavity
To verify theoretical analysis, a $400 \mathrm{GHz}$ second harmonic gyrotron with confocal cavity is has been manufactured and assembled in the UESTC. A photo of the confocal cavity are shown in fig. 5, and the photo of assembly prototype tube is presented in fig. 6 .

The prototype tube adopts a triode-type magnetroninjection gun (MIG) which developed for $220 \mathrm{GHz}$ gyrotron, and optimized for design parameters. The output from confocal cavity is converted from confocal waveguide to homocentric waveguide by an irregular quasi-optical taper, and transported into cylindrical waveguide.

The initial experiments have been performed based on a $9.2 \mathrm{~T}$ superconducting magnet. The accelerating voltage for MIG is provided by a high voltage modulator, which can supply about $120 \mathrm{kV}$ with a pulse width of $100 \mu$ s. The output power of gyrotron is measured by a modified laser calorimeter, amplified by a low-noiseamplifier (LNA), and recorded by a Data Acquisition (DAQ) card. The frequency was measured using two VivaTech harmonic mixers. One is used to detect fundamental output, and another is used to detect second harmonic output.

Under the operation point of maximum output power (40 kV, $4.75 \mathrm{~A}$, and $7.51 \mathrm{~T}$ ), the detected oscilloscope traces are shown in fig. 7(a) which shows the Beam voltage, collector current, IF signal. Figure4 (b) shows the expanded waveform of partial IF signal with a high sample rate of $8 \mathrm{GSa} / \mathrm{s}$. The initial experiment results show that the second harmonic output of $\mathrm{TE}_{0,11}$ mode and the fundamental output of $\mathrm{TE}_{0,6}$ mode are observed. The frequency of second harmonic output is $\sim 396 \mathrm{GHz}$ with $1.5 \mathrm{~kW}$ power. The results demonstrate that confocal cavity has good mode selectivity and would be used to develop high harmonic terahertz gyrotron.

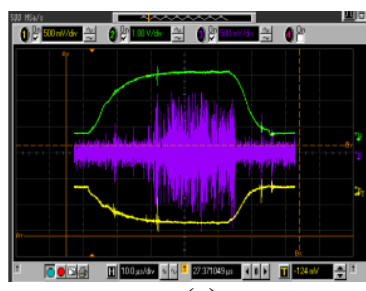

(a)

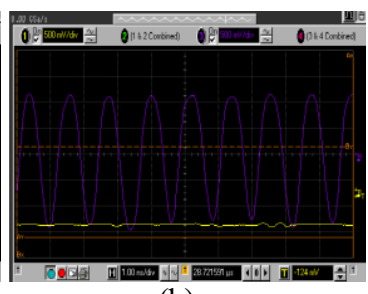

(b)
Fig. 7. Beam voltage, collector current, IF signal envelop and partial expanded IF signal waveform

This work is supported by the National Natural Science Foundation of China under Grant 61401064, and the CAEP THz Science and Technology Foundation under the Grant No. CAEPTHZ201403.

\section{References}

1. Hu, W., Shapiro, A., Kreischer, K. E., Temkin, R. J. 140$\mathrm{GHz}$ Gyrotron Experiments Based on a Confocal Cavity // IEEE Trans. Plasma Sci. 1998. V. 26, No. 3, PP. 366-374.

2. Sirigiri, J. R., Shapiro, M. A., Temkin, R. J. High-Power 140-GHz Quasioptical Gyrotron Traveling-Wave Amplifier // Phys. Rev. Lett. 2003. V. 90, No. 25, P. 258302.

3. Fu, $W$., Guan, $X$., Yan, Y. High harmonic terahertz confocal gyrotron with nonuniform electron beam // Physics of Plasmas 2016. V. 23, No. 1, P. 013301.

4. Fu, W., Guan, X., Chen, C., Li, X., Yuan X., Yan, Y. Design and Experiment of a 220/420-GHz Gyrotron for Nondestructive Evaluation // IEEE Trans. Electron Devices, 2014, V. 61, No. 7, PP. 2531-2537. 